\title{
Recorrido socioambiental por los territorios indígenas en la Amazonia venezolana
}

\author{
Socio-environmental path of indigenous territories in the Venezuelan \\ Amazon
}

\section{Abordagem socioambiental no estudo dos territórios indígenas na Amazônia venezuelana}

\author{
Melgris José Becerra \\ Universidade Federal do Pará, Instituto de Geociências. Belém do Pará - Brasil \\ jose.becerra.ruiz@gmail.com melgris.ruiz@iq.ufpa.br \\ https://orcid.org/0000-0001-6675-7370
}

\begin{abstract}
RESUMEN
La Amazonia venezolana es un territorio que necesita del trabajo de los profesionales de la geografía. Está región se ha caracterizado (en los libros) como una región inhóspita e insondable. Sin embargo, es un territorio lleno culturales ancestrales con prácticas territoriales importantes. En este texto se pretende demostrar que los conocimientos geográficos están desarrollados en las diferentes culturas y sociedades en ellas convergen los conocimientos propios que son clave para la gobernanza territorial y gestión del territorio. Se narra una historia de vida personal que clarifica, cuestiona y amalgama conocimientos aprendidos en la academia y experiencias locales con los pueblos indígenas de la amazonia venezolana. Finalmente, concluye que las experiencias enriquecen el conocimiento y son base para la comprender los territorios en donde confluyen diversas voces, matices y elementos cotidianos que van configurando el espacio geográfico local y la vida en la amazonia.
\end{abstract}

\begin{abstract}
The Venezuelan Amazon is a place that has not achieved a good capillarity among geography professionals in Venezuela. This region has been characterized (in books) as an inhospitable and unfathomable region. This text intends to demonstrate that geographic knowledge is developed in different cultures and societies in which their own knowledge converges and is key to territorial governance and management of the territory. It narrates a personal life story that clarifies, questions, and amalgamates knowledge learned in the academy and local experiences with the indigenous peoples of the Venezuelan Amazon. Finally, it concludes that experiences enrich knowledge and are the basis for understanding the territories where diverse voices, nuances and daily elements that shape the local geographic space and life in the Amazon converge.
\end{abstract}

\section{RESUMO}

A Amazónia venezuelana é um lugar que não alcançou uma boa capilaridade entre os profissionais da geografia na Venezuela. Esta região tem sido caracterizada (em livros) como uma região inóspita fías y ciencias sociales, 3(5), 85-92. https://dx.doi.org/10.15381/espiral.v3i5.20872 
e insondável. Este texto pretende demonstrar que o conhecimento geográfico é desenvolvido em diferentes culturas e sociedades, onde convergem os conhecimentos que são fundamentais para a governação e gestão territorial. Narra uma história de vida pessoal que esclarece, questiona e amalgama conhecimentos aprendidos na academia e experiências locais com os povos indígenas da Amazónia venezuelana. Finalmente, conclui que as experiências enriquecem o conhecimento e são a base para a compreensão dos territórios onde diversas vozes, nuances e elementos do quotidiano se juntam para moldar o espaço geográfico local e a vida na Amazónia.

PALABRAS CLAVE: Territorio; amazonia; Yekwana; Piaroa.

KEYWORDS: Territory; Amazon; Yekwana; Piaroa.

PALAVRAS-CHAVE: Território; Amazônia; Yekwana; Piaroa.

\section{Introducción}

Las actividades de los geógrafos están marcadas por una incesante búsqueda de nuevas formas de hacer geografía tanto en los ámbitos urbanos, rurales, industriales e incluso comerciales o de marketing. En mi caso, mi interés estuvo marcado por explorar los temas indígenas, en principio, por la curiosidad de conocer mis raíces, mi historia. Posteriormente, logré incorporar la visión geográfica.

Conocer sobre la geografía no está definido únicamente en los libros, también forma parte de la vivencia cotidiana y del trabajo con personas en sus comunidades desde el territorio. La territorialidad indígena es diversa, así como son diversos sus pueblos. Los pueblos indígenas que tienen un mayor contacto con la sociedad nacional, están en constante conflicto entre retomar sus prácticas y conocimientos ancestrales o incorporar elementos aprendidos como una forma de complementar sus conocimientos (Martin, 2017). No obstante, ese conocimiento híbrido logra una traducción e interlocución con la sociedad nacional.

El territorio es la herramienta principal del geógrafo y es allí donde ocurren todas las interacciones políticas, económicas, sociales y culturales, es decir, la vida. Estudiar el territorio amazónico invita a pensar en su estructura, su conformación socioespacial, ya que, es en este espacio donde confluyen y se alimentan diferentes formas de apropiación territorial donde convergen culturas y se alimenta la vida (Becerra, Flores Rangel, Gonçalves, \& Tovar, 2021). La apropiación del territorio amazónico se basa en una estructura de relaciones entre las sociedades amazónicas, que se expresan en sus intercambios territoriales. La utilización de los espacios para producción de alimentos, vivienda, ocio y placer, son garantía para su estabilidad social, pertenencia territorial y seguridad alimentaria (Levis et al., 2018). No obstante, el territorio indígena va mucho más allá, se adentran en energías, pensamientos, sentimientos y voluntades (Varese, 2020). Sus prácticas territoriales están profundamente arraigadas al mundo espiritual, y es desde esta perspectiva donde se reterritrorializa (Cooper, 2019), a manera de traducción, el espacio que navega entre el espacio físico y el mundo espiritual.

Esta bitácora tiene como objetivo demostrar que el aprendizaje geográfico está en el campo, entre las comunidades, entendiendo las configuraciones espaciales del territorio. El territorio sustenta a las sociedades que lo componen y son los individuos quienes se apropian y territorializan con sus vivencias. En primer lugar, relatamos hacia donde vamos, se desarrolla el contexto geográfico. En segundo lugar, explicamos las actividades realizadas. En tercer lugar, se describen las situaciones y eventos sociales encontradas en los territorios visitados. En cuanto lugar, las reflexiones y aprendizajes. 


\section{Planificando la ruta en el Amazonas Venezolano}

En esta bitácora quiero señalar mi experiencia en campo como geógrafo en la amazonia venezolana, en el cual tenía como fin generar un documento de análisis socioambiental relacionado con los territorios indígenas. Partimos de la ciudad de Puerto Ayacucho en el estado Amazonas de Venezuela hacia dos territorios indígenas, el primero en visitar fue el territorio Yekwana ubicado en el municipio Manapiare, el segundo fue el territorio Piaroa (Uwottuja) ubicado en el municipio Autana, ambos en el estado Amazonas (véase figura 1).

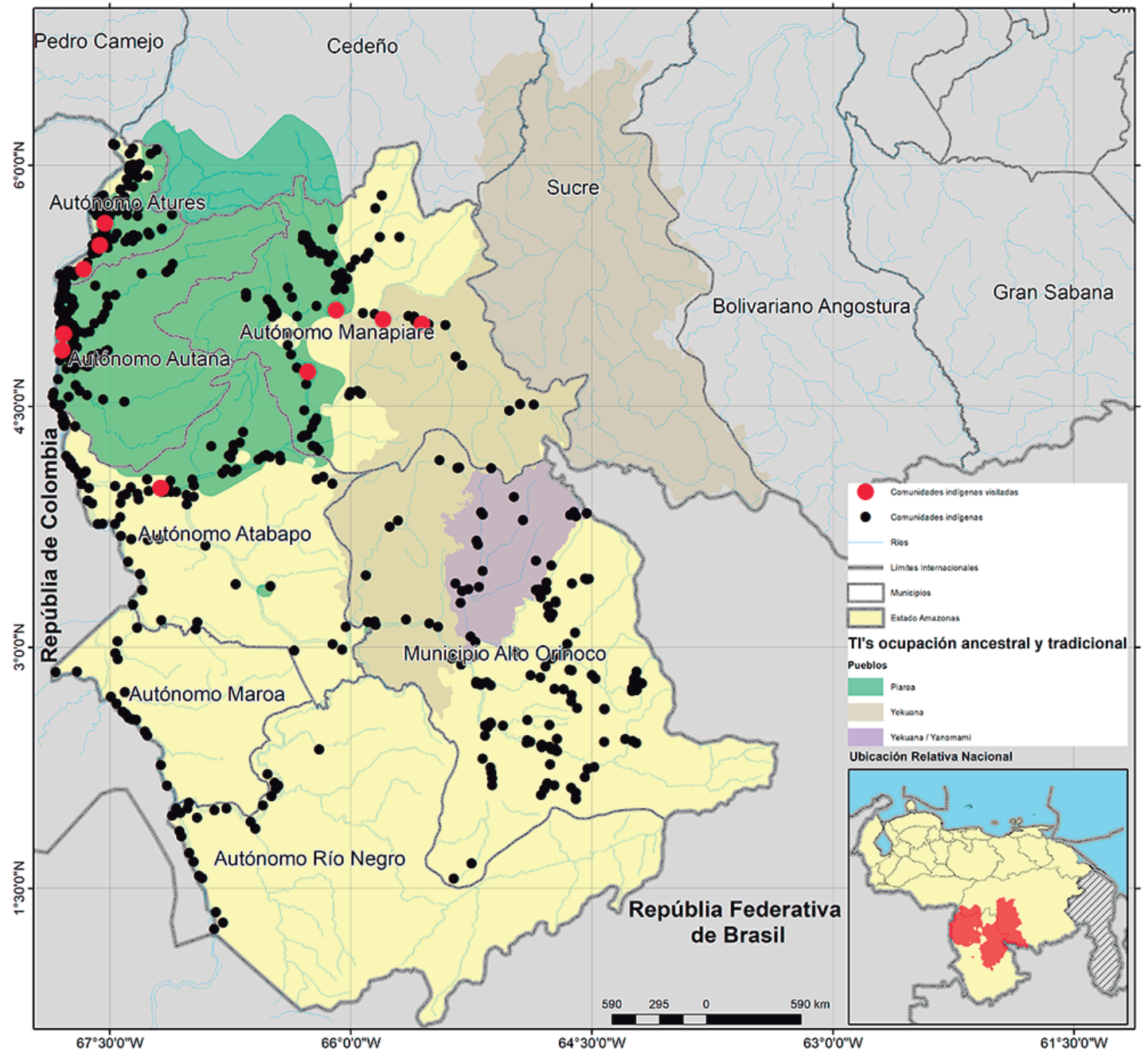

Figura 1. Territorio de ocupación ancestral y tradicional de los pueblos Yekwana y Piaroa (Uwottuja) en la amazonia venezolana.

Fuente: Elaboración propia con base en (RAISG, 2019, 2020)

La experiencia en dos territorios indígenas es enriquecedora, pues, los paisajes que habitan y sus interacciones territoriales y sociales son distintas. Cabe destacar, desde el punto de vista lingüístico cada pueblo tiene raíces diferentes y desde el punto de vista antropológico tiene una forma de constitución social propia, estos temas son muy interesantes y enriquece la visión del geógrafo, sin embargo, nos centraremos en el tema geográfico. Cada pueblo indígena tiene una apropiación diferenciada de los elementos que componen sus territorios desde la configuración del paisaje hasta la practicas ancestrales, culturales y aquellas prácticas adoptadas por la sociedad nacional.

El territorio de ocupación ancestral y tradicional del pueblo Yekwana es muy amplio. Este pueblo indígena se encuentra distribuido en la frontera entre Brasil y Venezuela 
ocupando aproximadamente 5 millones de hectáreas (Tillett, Senra, Becerra Ruiz, Saraiva Martins, \& Alves, 2014), sin embargo, de acuerdo con sus ancianos/sabedores el territorio puede extenderse hasta el océano atlántico, esto indica que el territorio de ocupación se ha sido reducido a pocos kilómetros. Su contacto con la sociedad nacional estuvo marcada por la fiebre del caucho y por el contacto con los misioneros, ambos contactos vinieron a influenciar la dinámica de asentamientos de este pueblo (ArveloJiménez, 1991). Es interesante la forma como se distribuyen las comunidades, estas, se ubican en una franja alargada que recorre el largo de sus ríos y meandros entre los ríos Caura y Venturi y Orinoco, esta ubicación tiene como objetivo resguardar los diferentes lugares sagrados tradicionales y culturales.

Por otro lado, el territorio ancestral del pueblo Piaroa (Uwottuja) está ubicado en la frontera entre Colombia y Venezuela con una superficie aproximada de 1.250.000 hectáreas (Becerra Ruiz, 2014), este pueblo ha mantenido su territorio de ocupación ancestral y tradicional a buen resguardo. Para evitar conflictos, este pueblo se refugia cada vez que su supervivencia se ve amenazada por el intercambio con la sociedad nacional, por tal razón, tienen varias casas que se ubicaban en lugares apartados, de modo que si tienen que movilizarse no afecta su capacidad de producción (Freire, 2003), en muchos casos estas se ubican en la cercanía de lugares sagrados y de respeto (véase figura 2). De acuerdo con los chamanes y sabios de las comunidades sus ancestros habitan las montañas, y cada montaña puede representar las casas de descanso, de culto, de aprendizaje (Rodd, 2018). Es allí donde los ancestros desde sus casas (montañas) les ofrecen los animales para la cacería, pesca, frutas, y es el lugar donde sus deidades están presentes (Morales, 2014). La distribución de sus comunidades ha sido en forma concéntrica, siempre alrededor del territorio sagrado compuesto por el macizo montañoso Cuao-Sipapo.

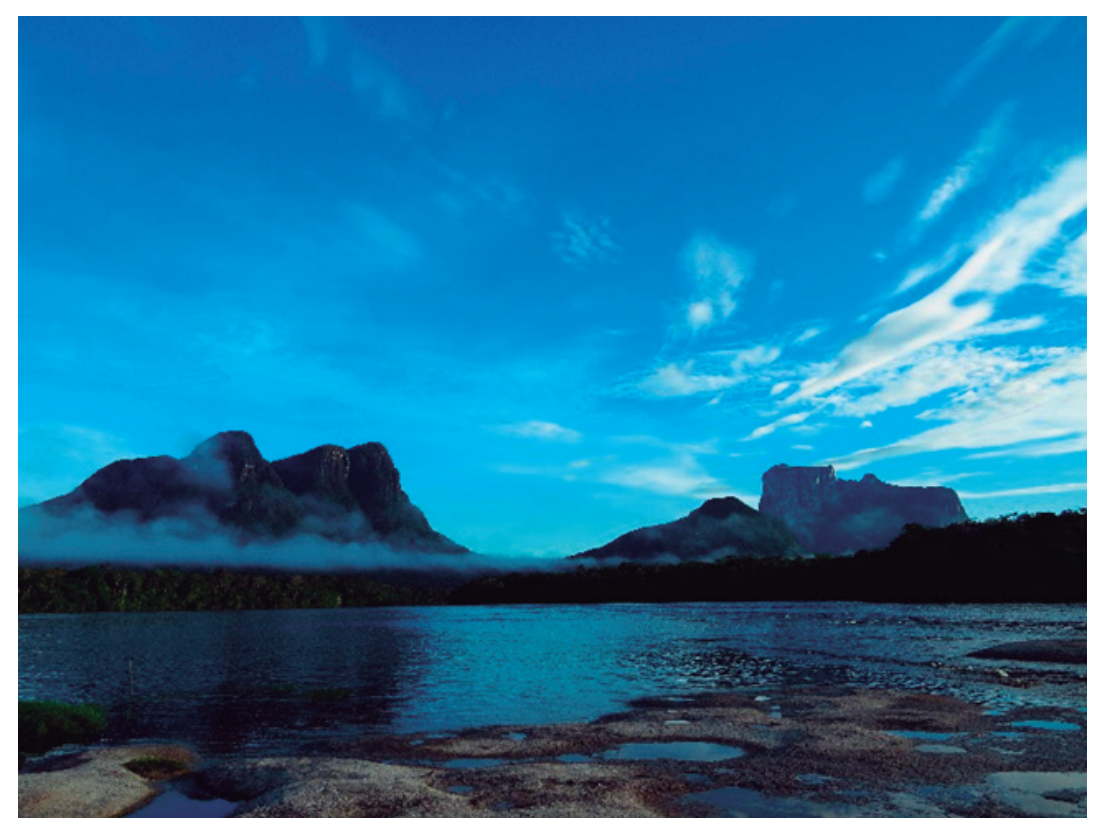

Figura 2. Montaña sagrada del pueblo Piaroa. A la izquierda casa de Chejeru, al fondo Cerro Autana (El árbol de la vida)

\section{Consideraciones para el trabajo en las comunidades indígenas}

Desde este viaje reconozco que existen muchas similitudes entre los pueblos indígenas que habitan la región amazónica, no obstante, existen diferencias significativas en cuanto a su forma de organización social, idiomas culturas y costumbres (Bremner \& Lu, 2006; Kearney \& Varese, 1995). Estas características permitieron redefinir mi concepto de territorio, conocer de primera mano como se construye, mantiene y defiende(n) los territorios para desarrollar la vida en 
comunidad. Cada pueblo ha consolidado diferentes estrategias para la preservación y defensa de los territorios donde habitan.

Al convivir en las comunidades es necesario cumplir con sus prácticas de convivencia que es igual para todos en las comunidades. Las actividades diarias garantizan el funcionamiento integral de la vida comunitaria. Estas actividades buscan sintonizar con las prácticas de las nuevas generaciones, pues, son aprendices del aprovechamiento diario de los recursos que se encuentran en sus territorios y futuros garantes de la protección territorial, sin embargo, en muchos casos los interese de los más jóvenes están alejados de las comunidades. Actividades como preparar el terreno para sembrar, preparar la semilla o tucos (palos recortados de aprox. $10 \mathrm{~cm}$ ) para la siembra de frutos y tubérculos, así como la cosecha. En estas acciones están definidas para cada individuo haciendo una cadena de labores productiva en un conuco.

Para el aprovechamiento de los recursos de sus conucos se deben sembrar distintas frutas como guama, piña, así como tubérculos ñame, batata, ocumo, entre ellos, la yuca. Este tubérculo se puede distinguir entre yuca amarga y dulce. Una vez procesada la yuca amarga puede obtenerse el casabe y/o el mañoco que es base de la dieta diaria de estos pueblos, así como, la catara que es un picante típico con base en el jugo que desprende la yuca amarga, este es acompañante importante en su dieta diaria, aunque la preparación del picante es distinta para cada pueblo indígena.

\section{Metodologías de trabajo etnocartográfico}

Durante las reuniones buscábamos conocer un poco más los territorios y la práctica territorial, así, logramos conocer que significa las actividades diarias de sobrevivencia. La pesca y caza representan una de las actividades más productivas para las comunidades, pues, es allí donde se fortalece el conocimiento del territorio (López, 2018). Por otro lado, existen lugares definidos para la caza y pesca, así como, aquellos lugares que son prohibidos por los sabios para practicar estas actividades. Sin embargo, hay lugares de respeto que son excepcionales, es decir, se debe pedir permiso por medio de oraciones para realizar cualquier actividad. Explicar sobre el uso del territorio y transmitir esta información a los más jóvenes forman parte del ritual que realizan los adultos, dando espacio para apropiarse del territorio. Desde estas prácticas se puede decir que los pueblos indígenas actúan dentro de su cosmovisión, esta, es inseparable de las relaciones y obligaciones socioculturales, políticas, legales y otras, basadas en gran medida en el territorio (Latulippe \& Klenk, 2020), es allí donde hay una enraizamiento cultural en los territorios.

Durante estos viajes hubo encuentros con distintos actores locales y funcionales que articulaban espacios de encuentro para las actividades que estábamos realizando, entre ellos, se encontraba la sabia del pueblo Piaroa, residente de una de las comunidades con las que estábamos trabajando. Ella condujo en su idioma buena parte de la reunión, no obstante, las veces que habló en español se refirió al territorio como parte de su casa o como una casa donde vivimos todos. Este discurso tiene una carga geográfica muy interesante, pues, esa afirmación vincula todo el conocimiento propio, entendiendo los elementos de espacio geográfico en su multidimensionalidad, no habla únicamente de la tierra, sino también habla del bosque, los ríos, las montañas y sus territorios espirituales, en esa afirmación está cargado todo su conocimiento ancestral (Aswani, Lemahieu, \& Sauer, 2018). Lo que permite reconocer que el conocimiento geográfico es vital para la protección territorial.

Posterior a las reuniones generales, conversamos con representantes de las comunidades para trabajar sobre varios temas vinculados a las formas de distribución territorial a través de mapas mentales, es decir, conocer los sitios donde realizaban diversas actividades referidas a su cotidianidad. Por ejemplo, sitios de pesca de un tipo de pescado, sitios de lombrices, cuevas donde viven los tigres, sabanas donde 
viven los jabalíes, selvas donde viven los monos, entre muchos otros lugares que para ellos son importantes en su vida cotidiana.

Otros territorios que visibilizaron fueron los sitios sagrados, en aquellas cadenas montañosas habitaban las deidades superiores, por su parte, en los piedemontes y zonas de colinas habitaban otros seres que protegían los territorios. Recuerdo que un sabio del pueblo Piaroa contó en algún momento sobre las zonas donde habitan los no humanos, esto significa, que habitan en el mundo espiritual, pero, que hacen vida como personas de carne y hueso (Becerra Ruiz, 2014). Parece un relato insólito, sin embargo, ellos afirman que son reales. Según su experiencia, se pueden ver en lugares dentro de la selva señales de presencia humana como fogones para la cocción de alimentos aun encendidos, sin embargo, no hay presencia humana en esos lugares.

\section{Contexto socioambiental y amenazas latentes}

Durante este estudio, se evidencio que hay amenazas y presiones que atentan contra la estabilidad de los pueblos indígenas. En este sentido, los territorios de la amazonia venezolana se han visto amenazados por prácticas ilegales para la extracción de recursos minerales (RAISG, 2012, 2019), siendo esta práctica motivo para desplazarse de sus territorios de ocupación tradicional hacia sus lugares ancestrales (Tigre, 2017). Estas prácticas de volver a su territorio se realizan cada vez que se ve amenazada su supervivencia física, cultural y espiritual como pueblo. Se desplazan desde los lugares donde hacen su vida dentro de la sociedad nacional para mantener, preservar y resguardar el corazón de su cultura desde idiomas, tradiciones hasta sus prácticas culturales y ancestrales.

Estas formas de agrupamiento de las comunidades y como utilizan el territorio, es adecuada dada la experiencia y la vivencia en estoslugares. Senos ocurrió una pregunta que parece obvia, sin embargo, tiene mucho sentido para quien no está inmiscuido en estas prácticas indígenas y geográficas. Pero ¿Por qué los pueblos indígenas Yekwana y Piaroa (Uwottuja) se resguardan en estos territorios y no en otro? Considerando que sus territorios son tan amplios y ocupan cientos de kilómetros. Porque es allí donde reposa el corazón de su cultura, donde están sus ancestros, el lugar donde reposa la vida tradicional y cultural que garantizan su supervivencia. Forma parte de una estrategia utilizada para preservar sus lugares sagrados, garantizar su hábitat y respetar la vida.

Esto significa quelas actividades para aprovechar los recursos del territorio representan una forma de vida, asociado al significado de territorio, sociedad y familia. Estas acciones no solo están relacionadas con el trabajo, también redundan, en sabores, olores y texturas que resignifican el espacio y promueve un arraigo cultural significativo (Vieco, Franky, \& Echeverri, 2000). Estos elementos son la garantía de la protección de los territorios, la cual, está asociada al auto reconocimiento y reconocimiento de culturas con raíces ancestrales, la cual, reposa en los territorios(Becerra et al., 2021).

\section{Reflexiones finales}

Explorando el territorio amazónico podemos encontrar que el conocimiento territorial, es una forma innata de las sociedades y son necesarios en contextos urbanos, rurales, en este caso, en la amazonia venezolana sirve para impulsar procesos de gobernanza territorial, gestionar sus territorios e incidir ante los organismos del Estado con información de tipo cartográfica, referencial y cultural como base para garantizar el reconocimiento de sus territorios de ocupación ancestral.

Los territorios indígenas se encuentran amenazados por un incremento significativo de las actividades extractivas, las cuales han llevado al aumento significativo de enfermedades erradicadas (Grillet et al., 2019), la minería ilegal y existen serias 
presiones sobre estos territorios para la explotación de los recursos minerales, ya en los límites con el estado Amazonas en el estado Bolívar (Benfica, Hernández, \& Becerra Ruiz, 2017). El Estado nacional implementó el denominado Arco Minero del Orinoco que tiene fuertes impactos en el territorio indígena por el desplazamiento de actores mineros a regiones con poca vocación minera (Ruiz, 2018). Los pueblos indígenas se han visto presa de estas actividades y muchos se han vinculado con la explotación de oro, otros forman parte de la trama de distribución de combustibles, alimentos, medicinas e incluso en la trata de blancas (A. Acosta, 2011; A. M. Acosta, 2017). El complejo entramado socioambiental de la amazonia evidencia que están en riesgo las comunidades, sus territorios y la casa común.

Ciertamente, las amenazas sobre los territorios indígenas se han incrementado en los últimos años, esto ha permitido que muchos actores armados, legales, ilegales y paralegales se hayan instalado en los territorios por cooptación, coacción o por la fuerza, sin embargo, estas situaciones no invisibilizan las estrategias que subyacen en las prácticas de los individuos y las sociedades en los territorios indígenas.

Para finalizar les puedo decir que el análisis socioambiental de la amazonia venezolana tuvo un peso importante en la comprensión del concepto territorio. Contrastar experiencias, lecciones y aprendizajes estimula el interés de conocer estas interacciones en el ámbito humano, social, económico, político, cultural que además tiene una relación directa con el manejo de los recursos naturales, las relaciones de poder, así como, el mundo espiritual indígena, aún poco conocido en geografía.

\section{Referencias Bibliográficas}

Acosta, A. (2011). Extractivismo y neoextractivismo: Dos caras de la misma maldición. Más allá del desarrollo, 1, 83-118.

Acosta, A. M. (2017). Oro, sexo y poder: Violencia contra las mujeres indígenas en los contextos mineros de la frontera amazónica colombo-venezolana. Textos e Debates, 1(31). https://doi. org/10.18227/2217-1448ted.v1i31.4256

Arvelo-Jiménez, N. (1991). Indigenismo y debate sobre desarrollo amazónico: Reflexiones a partir de la experiencia venezolana ( $1 .^{\text {a }}$ ed.). Brasilia: UnB.

Aswani, S., Lemahieu, A., \& Sauer, W. H. H. (2018). Global trends of local ecological knowledge and future implications. PLOS ONE, 13(4), e0195440. https://doi.org/10.1371/journal. pone. 0195440

Becerra, M. J., Flores Rangel, J. A., Gonçalves, C. U., \& Tovar, G. I. (2021). The Indigenous Territories and Local Sustainable Development in the Amazon Region. En R. B. Singh, S. Chatterjee, M. Mishra, \& A. J. de Lucena (Eds.), Practices in Regional Science and Sustainable Regional Development: Experiences from the Global South (pp. 69-112). Singapore: Springer. https://doi.org/10.1007/978-981-16-2221-2_4

Becerra Ruiz, J. (2014). Mapa Territorio Tearime Sirikoi Aerime Suititi. Deiyu Wachurijua (Pueblo en aislamiento voluntario) sector Sipapo [Temático]. En Teärime Siri'koi Aerime Suititi El Territorio Uwottüja (1. ${ }^{a}$ ed.). Bogotá, Colombia: OIPUS-WATANIBA. Recuperado de https://www. iwgia.org/images/publications/0710_UOTTUJA_LIBRO_eb.pdf

Benfica, E., Hernández, M., \& Becerra Ruiz, J. (2017). Garimpo ilegal nos Territorios Yanomami e Ye'kwana (Brasil-Venezuela). Rede de Informação Geográfica Binacional Yanomami e Ye'kwana [Temática]. Sao Paulo, Brasil: ISA. Recuperado de https://acervo.socioambiental.org/ acervo/mapas-e-cartas-topograficas/bacia-do-rio-negro/garimpo-ilegal-nos-territoriosyanomami-e

Bremner, J., \& Lu, F. (2006). Common Property among Indigenous Peoples of the Ecuadorian Amazon. Conservation and Society, 4(4), 499-521.

Cooper, D. G. (2019). The spiritual geography of landscape. 16. 
Freire, G. (2003). Tradition, Change and Land Rights: Land Use and Territorial Strategies among the Piaroa. Critique of Anthropology, 23(4), 349-372. https://doi. org/10.1177/0308275X03234006

Grillet, M. E., Hernández-Villena, J. V., Llewellyn, M. S., Paniz-Mondolfi, A. E., Tami, A., Vincenti-Gonzalez, M. F., ... Alarcón-de Noya, B. (2019). Venezuela's humanitarian crisis, resurgence of vector-borne diseases, and implications for spillover in the region. The Lancet Infectious Diseases, 19(5), e149-e161. https://doi.org/10.1016/S1473-3099(18)30757-6

Kearney, M., \& Varese, S. (1995). Latin America's Indigenous Peoples: Changing Identities and Forms of Resistance. En Capital, Power, and Inequality in Latin America. Routledge.

Latulippe, N., \& Klenk, N. (2020). Making room and moving over: Knowledge co-production, Indigenous knowledge sovereignty and the politics of global environmental change decision-making. Current Opinion in Environmental Sustainability, 42, 7-14. https://doi. org/10.1016/j.cosust.2019.10.010

Levis, C., Flores, B. M., Moreira, P. A., Luize, B. G., Alves, R. P., Franco-Moraes, J., ... Clement, C. R. (2018). How People Domesticated Amazonian Forests. Frontiers in Ecology and Evolution, 0. https://doi.org/10.3389/fevo.2017.00171

López, M. G. H. (2018). Fortalecer la territorialidad en las comunidades. Universidad en Diálogo: Revista de Extensión, 8(2), 51-69. https://doi.org/10.15359/udre.8-2.4

Martin, B. (2017). Methodology is content: Indigenous approaches to research and knowledge. Educational Philosophy and Theory, 49(14), 1392-1400. https://doi.org/10.1080/00131857.2017 .1298034

Morales, C. (2014). Identidad del creador y el origen Uwottüja. En C. Morales \& M. Quispe (Eds.), Teärime Siri'koi Aerime Suititi El Territorio Uwottüja (1. ${ }^{a}$ ed., pp. 19-40). Bogotá, Colombia: OIPUS-WATANIBA.

RAISG. (2012). Amazonía bajo presión (1. ${ }^{a}$ ed.). Sao Paulo, Brasil: Instituto Socioambiental. Recuperado de https://www.amazoniasocioambiental.org/es/publicacion/amazoniabajo-presion/

RAISG. (2019). Amazonía 2019 Áreas protegidas, Territorios indígenas. [Temático]. Sao Paulo: RAISG. Recuperado de https://www.amazoniasocioambiental.org/es/mapas/

RAISG. (2020). Datos cartográficos [Organización]. Recuperado 1 de julio de 2020, de RAISG website: https://www.amazoniasocioambiental.org/es/mapas/

Rodd, R. (2018). Piaroa Shamanic Ethics and Ethos: Living by the Law and the Good Life of Tranquillity. International Journal of Latin American Religions, 2(2), 315-333. https://doi. org/10.1007/s41603-018-0059-0

Ruiz, F. J. (2018). El Arco Minero del Orinoco Diversificación del extractivismo y nuevos regímenes biopolíticos. Nueva Sociedad, (274), 129-141.

Tigre, M. A. (2017). Threats to the Amazon Rainforest: Deforestation and Climate Change. En Regional Cooperation in Amazonia (pp. 48-75). Brill Nijhoff. https://doi. org/10.1163/9789004313507_004

Tillett, A., Senra, E. B., Becerra Ruiz, J., Saraiva Martins, M., \& Alves, R. (2014). Território e Comunidades Yanomami Brasil-Venezuela 2014 [Temático]. Sao Paulo, Brasil: ISA. Recuperado de https://acervo.socioambiental.org/acervo/mapas-e-cartas-topograficas/bacia-do-rionegro/territorio-e-comunidades-yanomami-brasil

Varese, S. (2020). Ethical Cosmologies in Amazonia. Mester, 49(1). https://doi.org/10.5070/ M3491051392

Vieco, J. J., Franky, C. E., \& Echeverri, J. A. (Eds.). (2000). Territorialidad indígena y ordenamiento en la Amazonia (1. ${ }^{a}$ ed.). Leticia: UNIBIBLOS. Recuperado de https://www.academia. edu/6686571/Territorialidad_ind\%C3\%ADgena_y_ordenamiento_en_la_Amazonia 